MATHEMATICS OF COMPUTATION

Volume 67, Number 224, October 1998, Pages 1601-1616

S 0025-5718(98)00976-4

\title{
ASYMPTOTIC UPPER BOUNDS FOR THE COEFFICIENTS IN THE CHEBYSHEV SERIES EXPANSION FOR A GENERAL ORDER INTEGRAL OF A FUNCTION
}

\author{
NATASHA FLYER
}

\begin{abstract}
The usual way to determine the asymptotic behavior of the Chebyshev coefficients for a function is to apply the method of steepest descent to the integral representation of the coefficients. However, the procedure is usually laborious. We prove an asymptotic upper bound on the Chebyshev coefficients for the $k^{t h}$ integral of a function. The tightness of this upper bound is then analyzed for the case $k=1$, the first integral of a function. It is shown that for geometrically converging Chebyshev series the theorem gives the tightest upper bound possible as $n \rightarrow \infty$. For functions that are singular at the endpoints of the Chebyshev interval, $x= \pm 1$, the theorem is weakened. Two examples are given. In the first example, we apply the method of steepest descent to directly determine (laboriously!) the asymptotic Chebyshev coefficients for a function whose asymptotics have not been given previously in the literature: a Gaussian with a maximum at an endpoint of the expansion interval. We then easily obtain the asymptotic behavior of its first integral, the error function, through the application of the theorem. The second example shows the theorem is weakened for functions that are regular except at $x= \pm 1$. We conjecture that it is only for this class of functions that the theorem gives a poor upper bound.
\end{abstract}

\section{INTRODUCTION}

Determining the rate of convergence for the Chebyshev expansion of a function

$$
f(x)=\sum_{n=0}^{\infty} a_{n} T_{n}(x)
$$

as $n \rightarrow \infty$ requires an asymptotic approximation for the coefficients, $a_{n}$, when an exact analytical form is not known. These approximations are usually obtained by applying the method of steepest descent to the integral representation of the coefficients. This procedure, though, can be lengthy and involved. However, if the function we are expanding is an integral of a function whose Chebyshev coefficients are already known, either exactly or asymptotically, then there should be a relation between the known coefficients and those for the integral. For instance, it is known

Received by the editor September 20, 1996 and, in revised form, March 3, 1997.

1991 Mathematics Subject Classification. Primary 42C10.

Key words and phrases. Chebyshev series.

The author would like to express her deep appreciation and gratitude for the valuable discussions and comments of Prof. John P. Boyd at the University of Michigan. This work was supported by NASA Grant NGT-51409. 
that if a function is expanded in a Fourier series, then the coefficients of the $k^{\text {th }}$ integral of the function are just the original coefficients multiplied by $\frac{1}{(i n)^{k}}$.

It is the aim of this paper to show that a similar relation exists for the Chebyshev case. Even though an exact formula for the Chebyshev coefficients of the $k^{\text {th }}$ integral of a function are derived, due to its complicated nature we retreat to an upper bound on the coefficients in order to get a simple interpretation of the formula. We begin by proving a theorem that calculates recursively the coefficients for the $k^{t h}$ integral of the Chebyshev polynomial $T_{n}(x)$. This result is then used to derive a second theorem for calculating these coefficients directly. From Theorem 2.1, a corollary is proved that shows the asymptotic behavior for large $n$ of the coefficients for the $k^{t h}$ integral of a Chebyshev polynomial. Then these allow us to define the main theorem (Theorem 4.1) of this paper which gives an upper bound to the Chebyshev coefficients of the $k^{\text {th }}$ integral of a function. The next order of business is to determine when does the theorem not give the tightest upper bound possible. In order to answer this question, for the rest of the paper we consider the case $k=1$, the first integral of a function. We then define a criterion that needs to be met in order for Theorem 4.1 to give a poor upper bound. The criterion essentially states that if two Chebyshev coefficients whose indices differ by two (e.g. $a_{n-1}$ and $\left.a_{n+1}\right)$ cancel when subtracted, in the limit as $n \rightarrow \infty$, Theorem 4.1 gives a poor upper bound. It is shown for a function whose Chebyshev series is converging at a geometric rate, the usual rate of convergence for Chebyshev series, that the coefficients do not meet the criterion and the theorem gives the tightest upper bound possible for the coefficients of the first integral of the function.

Two examples illustrate the concepts. The first example illustrates that the theorem is much easier than the method of steepest descent. The method of steepest descent is applied, laboriously, to directly determine the asymptotic Chebyshev coefficients of a Gaussian with a maximum at an endpoint of the Chebyshev interval. This function was chosen since the asymptotics of its Chebyshev coefficients has not been given previously in the literature. In contrast, obtaining the coefficients of the error function, which is the first integral of the the Gaussian, is almost trivial through Theorem 4.1. The second example illustrates a class of functions where the theorem gives a poor upper bound: functions that are regular everywhere on the Chebyshev expansion interval $x \epsilon[-1,1]$ except at the endpoints.

\section{AN UPPER BOUND FOR THE COEFFICIENTS OF THE $k^{\text {th }}$ INTEGRAL OF A CheBYShev POLYNOMIAL}

The recurrence relation for calculating the Chebyshev coefficients for the first integral of a function is well established and given in texts such as [9]. The following theorem generalizes on that relation, providing the coefficients for a general order integral.

Theorem 2.1. The $k^{\text {th }}$ integral of the Chebyshev polynomial, $T_{n}(x)$, is given by

$$
\int^{(k)} T_{n}(x) d^{k} x=\left(\frac{1}{2}\right)^{k} \sum_{i=0}^{k}(-1)^{i} a_{k, i}^{(n)} T_{n+k-2 i}(x), \quad n>k,
$$

where $a_{k, i}^{(n)}$ can be calculated recursively by

$$
a_{k, i}^{(n)}=\frac{1}{n+k-2 i}\left(a_{k-1, i-1}^{(n)}+a_{k-1, i}^{(n)}\right), \quad 0<i<k,
$$


with

$$
a_{k, 0}^{(n)}=\frac{n !}{(n+k) !}, \quad a_{k, k}^{(n)}=\frac{(n-k-1) !}{(n-1) !} .
$$

Proof. We prove the result by induction. We first show the theorem holds for $\mathrm{k}=1$. Using the Chebyshev to Fourier cosine transformation, $x=\cos (t)$, and then a trigonometric identity, we have

$$
\int \cos (n t) \sin (t) d t=\frac{1}{2}\left(\frac{\cos (n+1) t}{n+1}-\frac{\cos (n-1) t}{n-1}\right) .
$$

It can be seen that Equation 2.3 is the same as Equation 2.1 for $k=1$.

Now, assume Theorem 2.1 is true for arbitrary $k$. Then,

$$
\left(\frac{1}{2}\right)^{k} \sum_{i=0}^{k}(-1)^{i} a_{k, i}^{(n)} \int \cos ((n+k-2 i) t) \sin (t) d t
$$

$$
\begin{gathered}
=\left(\frac{1}{2}\right)^{k+1} \sum_{i=0}^{k}(-1)^{i} a_{k, i}^{(n)}\left[\frac{\cos ((n+k+1-2 i) t)}{n+k+1-2 i}-\frac{\cos ((n+k-1-2 i) t)}{n+k-1-2 i}\right] \\
=\left(\frac{1}{2}\right)^{k+1}\left[a_{k, 0}^{(n)}\left[\frac{\cos ((n+k+1) t)}{n+k+1}-\frac{\cos ((n+k-1) t)}{n+k-1}\right]\right. \\
-a_{k, 1}^{(n)}\left[\frac{\cos ((n+k-1) t)}{n+k-1}-\frac{\cos ((n+k-3) t)}{n+k-3}\right] \\
+a_{k, 2}^{(n)}\left[\frac{\cos ((n+k-3) t)}{n+k-3}-\frac{\cos ((n+k-5) t)}{n+k-5}\right]-\cdots \\
\left.+a_{k, k}^{(n)}\left[\frac{\cos ((n-k+1) t)}{n-k+1}-\frac{\cos ((n-k-1) t)}{n-k-1}\right]\right]
\end{gathered}
$$

$$
\begin{aligned}
=\left(\frac{1}{2}\right)^{k+1}[ & \frac{a_{k, 0}^{(n)}}{n+k+1} \cos ((n+k+1) t) \\
& -\frac{1}{n+k-1}\left(a_{k, 0}^{(n)}+a_{k, 1}^{(n)}\right) \cos ((n+k-1) t) \\
& +\frac{1}{n+k-3}\left(a_{k, 1}^{(n)}+a_{k, 2}^{(n)}\right) \cos ((n+k-3) t)-\cdots \\
& \left.+\frac{a_{k, k}^{(n)}}{n-k-1} \cos ((n-k-1) t)\right] .
\end{aligned}
$$

However using (2.2), (2.7) is just

$$
\begin{gathered}
\left(\frac{1}{2}\right)^{k+1}\left[a_{k+1,0}^{(n)} \cos ((n+k+1) t)-a_{k+1,1}^{(n)} \cos ((n+k-1) t)\right. \\
\left.+a_{k+1,2}^{(n)} \cos ((n+k-3) t)-\cdots+a_{k+1, k}^{(n)} \cos ((n-k-1) t)\right] \\
=\left(\frac{1}{2}\right)^{k+1} \sum_{i=0}^{k+1}(-1)^{i} a_{k+1, i}^{(n)} \cos ((n+k+1-2 i) t) .
\end{gathered}
$$


The following corollary gives the asymptotic behavior of the coefficients $a_{k, i}^{(n)}$ as $n \rightarrow \infty$ for fixed $k$.

Corollary 2.2. A direct result of Theorem 2.1 is that the absolute value of the coefficients, $a_{k, i}^{(n)}, i=0, \ldots, k$, of the $k^{\text {th }}$ integral of the Chebyshev polynomial $T_{n}(x)$, are bounded from above by

$$
\left|a_{k, i}^{(n)}\right| \leq \frac{\left(\begin{array}{c}
k \\
i
\end{array}\right)}{n^{k}}\left(1+\varepsilon_{n}\right) \quad \text { as } n \rightarrow \infty \text { for fixed } k
$$

where $\varepsilon_{n}=O\left(\frac{1}{n}\right)>0$.

Proof. We will first show that the relation

$$
a_{k, i}^{(n)}=\frac{\left(\begin{array}{c}
k \\
i
\end{array}\right)}{n^{k}}+O\left(\frac{1}{n^{k+1}}\right)
$$

holds by induction and use of Theorem 2.1. For $k=1$, (2.2) gives

$$
\begin{array}{rlrl}
a_{1,0}^{(n)} & =\frac{1}{n+1} \quad \text { and } \quad a_{1,1}^{(n)} & =\frac{1}{n-1} \\
& =\frac{1}{n}+O\left(\frac{1}{n^{2}}\right) \quad \text { and } & a_{1,1}^{(n)} & =\frac{1}{n}+O\left(\frac{1}{n^{2}}\right)
\end{array}
$$

Since $i \leq k,(2.9)$ gives

$$
a_{1, i}^{(n)}=\frac{1}{n}+O\left(\frac{1}{n^{2}}\right)
$$

which agrees exactly with (2.10). Now assume (2.9) holds for arbitrary $k$, then by the recurrence relation given in $(2.2)$ we have

$$
\begin{aligned}
a_{k+1, i}^{(n)} & =\frac{1}{n+(k+1)-2 i}\left(a_{k, i-1}+a_{k, i}\right) \\
& =\frac{1}{n+(k+1)-2 i}\left(\frac{\left(\begin{array}{c}
k \\
i-1
\end{array}\right)}{n^{k}}+\frac{\left(\begin{array}{c}
k \\
i
\end{array}\right)}{n^{k}}+O\left(\frac{1}{n^{k+1}}\right)\right) \\
& =\frac{1}{n+(k+1)-2 i}\left(\frac{\left(\begin{array}{c}
k+1 \\
i
\end{array}\right)}{n^{k}}+O\left(\frac{1}{n^{k+1}}\right)\right) \\
a_{k+1, i}^{(n)} & =\frac{\left(\begin{array}{c}
k+1 \\
i
\end{array}\right)}{n^{k+1}}+O\left(\frac{1}{n^{k+2}}\right)
\end{aligned}
$$

The inequality in (2.8) is achieved by applying the triangle inequality to (2.15)

\section{The optimal EnVElope FUnCtion}

Before we can state the main theorem of the paper we need to define an optimal envelope function. The usefulness of such a definition is that for Chebyshev coefficients which oscillate (e.g. $\cos (f(n)))$, only the rate of decay is numerically important in terms of determining how Chebyshev polynomials are needed to reach 
a given accuracy. Therefore, it is advantageous to introduce a definition, the optimal envelope function, which filters out the oscillation and captures the asymptotic behavior of the coefficients that is numerically significant.

Definition 3.1 (Envelope function). A function $E\left(n ;\left\{b_{n}\right\}\right)$ is an envelope function for the spectral coefficients $b_{n}$ if $E\left(n ;\left\{b_{n}\right\}\right)$ is a positive monotonically decreasing function providing an upper bound to $\left\{b_{n}\right\}$ such that

1. $E\left(n ;\left\{b_{n}\right\}\right) \geq 0\{n: n \in N\}$,

2. $E\left(n ;\left\{b_{n}\right\}\right) \geq E\left(n+1 ;\left\{b_{n}\right\}\right)$,

3. $E\left(n ;\left\{b_{n}\right\}\right) \geq\left|b_{n}\right|$ for all $n$,

where $\mathrm{N}$ is the set of natural numbers.

Definition 3.2 (Optimal envelope function). A function $E_{o p t}\left(n ;\left\{b_{n}\right\}\right)$ is an optimal envelope function for the spectral coefficients $b_{n}$ if (i) $E_{o p t}\left(n ;\left\{b_{n}\right\}\right)$ is an envelope function and (ii) given any $\delta>0$, there exists an unbounded set of values for $n$ such that

$$
E_{\text {opt }}(n)-\left|b_{n}\right|<\delta
$$

From now on, we will denote $E_{\text {opt }}(n)$ simply by $E(n)$. It is important to note that an optimal envelope function always exists, for any convergent Chebyshev series, as is shown by the explicit construction

$$
E(n) \equiv \max _{j \geq n}\left|b_{j}\right| .
$$

However, the optimal envelope function as given by the definition is not unique. Steepest decent analysis, as presented later, often provides a smoother optimal envelope function than (3.2), which descends as a series of step functions like a flight of stairs. The shape of $E(n)$ does not matter, though, as long as it captures the general trend of the decrease in the coefficients, showing the asymptotic rate of convergence for the Chebyshev series.

\section{An upPer bound for the Chebyshev COEFFicients FOR A GENERAL ORDER INTEGRAL OF A FUNCTION}

The following is the main theorem of the paper, giving an upper bound on the Chebyshev coefficients of the $k^{\text {th }}$ integral of a function for large $n$.

Theorem 4.1. If $E\left(n ;\left\{b_{n}\right\}\right)$ is the optimal envelope function for the Chebyshev coefficients of a function $f(x)$, then the Chebyshev coefficients of the $k^{\text {th }}$ integral of $f(x)$,

$$
\int^{(1)} \cdots \int^{(k)} f(x) d^{k} x=\sum_{n=0}^{\infty} c_{n} T_{n}(x),
$$

are bounded from above as $n \rightarrow \infty$ for fixed $k$ by

$$
\left|c_{n}\right| \leq \frac{\left(1+\varepsilon_{n}\right)}{(2 n)^{k}}(k+1)\left(\begin{array}{c}
k \\
\frac{k}{2} \text { or } \frac{k-1}{2}
\end{array}\right) E(n-k),
$$

where $\varepsilon_{n}=O\left(\frac{1}{n}\right)>0$ and $\frac{k}{2}$ holds for $k$ even and $\frac{k-1}{2}$ holds for $k$ odd in the combinatorial term. 
Proof.

$$
\int^{(1)} \cdots \int^{(k)} f(x) d^{k} x=\sum_{n=0}^{\infty} c_{n} T_{n}(x)=\sum_{n=0}^{\infty} b_{n} \int^{(1)} \cdots \int^{(k)} T_{n}(x) d^{k} x .
$$

Using (2.1), (4.3) becomes

$$
\begin{aligned}
\int^{(k)} f(x) d^{k} x & =b_{0} \int^{(k)} T_{0} d^{k} x+\cdots+b_{k} \int^{(k)} T_{k} d^{k} x \\
& +\sum_{n=k+1}^{\infty} b_{n}\left\{\left(\frac{1}{2}\right)^{k} \sum_{i=0}^{k}(-1)^{i} a_{k, i}^{(n)} T_{n+k-2 i}(x)\right\} \\
& =b_{0} \int^{(k)} T_{0} d x+\cdots+b_{k} \int^{(k)} T_{k} d^{k} x \\
& +\sum_{n=1}^{2}\left\{\frac{(-1)^{k}}{2^{k}} \sum_{i=0}^{0}(-1)^{i} b_{n+k-2 i} a_{k, k-i}^{(n+k-2 i)}\right\} T_{n}(x) \\
& +\sum_{n=3}^{4}\left\{\frac{(-1)^{k}}{2^{k}} \sum_{i=0}^{1}(-1)^{i} b_{n+k-2 i} a_{k, k-i}^{(n+k-2 i)}\right\} T_{n}(x) \\
& +\cdots+\sum_{n=2 k-1}^{2 k}\left\{\frac{(-1)^{k}}{2^{k}} \sum_{i=0}^{k-1}(-1)^{i} b_{n+k-2 i} a_{k, k-i}^{(n+k-2 i)}\right\} T_{n}(x) \\
& +\sum_{n=2 k+1}^{\infty}\left\{\frac{(-1)^{k}}{2^{k}} \sum_{i=0}^{k}(-1)^{i} b_{n+k-2 i} a_{k, k-i}^{(n+k-2 i)}\right\} T_{n}(x) .
\end{aligned}
$$

For $n \geq 2 k+1, c_{n}$ is

$$
\begin{aligned}
c_{n} & =\frac{(-1)^{k}}{2^{k}} \sum_{i=0}^{k}(-1)^{i} b_{n+k-2 i} a_{k, k-i}^{(n+k-2 i)} \\
\left|c_{n}\right| & \leq\left|\frac{1}{2^{k}}\right| \sum_{i=0}^{k}\left|b_{n+k-2 i}\right|\left|a_{k, k-i}^{(n+k-2 i)}\right| .
\end{aligned}
$$

Given that $\left|b_{n+k-2 i}\right| \leq E(n+k-2 i)$, then

$$
\left|c_{n}\right| \leq\left|\frac{1}{2^{k}}\right| \sum_{i=0}^{k} E(n+k-2 i)\left|a_{k, k-i}^{(n+k-2 i)}\right| .
$$

We proved earlier in Corollary 2.2 that $\left|a_{k, i}^{(n)}\right| \leq \frac{\left(\begin{array}{c}k \\ i\end{array}\right)}{n^{k}}\left(1+\varepsilon_{n}\right)$ as $n \rightarrow \infty$ for fixed $k$. Thus,

$$
\begin{aligned}
& \left|c_{n}\right| \leq \frac{\left(1+\varepsilon_{n}\right)}{(2 n)^{k}} \sum_{i=0}^{k}\left(\begin{array}{c}
k \\
k-i
\end{array}\right) E(n+k-2 i), \\
& \left|c_{n}\right| \leq \frac{\left(1+\varepsilon_{n}\right)}{(2 n)^{k}}(k+1)\left(\begin{array}{c}
k \\
\frac{k}{2} \text { or } \frac{k-1}{2}
\end{array}\right) E(n-k),
\end{aligned}
$$

where $\frac{k}{2}$ holds for $k$ even and $\frac{k-1}{2}$ holds for $k$ odd in the combinatorial term. 
Although Theorem 4.1 gives an upper bound for the Chebyshev coefficients of the $k^{\text {th }}$ integral of a function, we can then ask the question how tight is this upper bound? If the types of functions can be identified for which Theorem 4.1 gives the optimal envelope function as $n \rightarrow \infty$, then the theorem gives an asymptotic expression for the tightest upper bound possible. In the remaining part of this section, it is proved for the case $k=1$, the first integral of a function, that if the Chebyshev coefficients for a function converge geometrically (refer to [6], Chapter 2) then Theorem 4.1 gives the optimal envelope function for the Chebyshev coefficients of the first integral of the function as $n \rightarrow \infty$. However, before this statement can be proved the following terms need to be defined.

Definition 4.2 (Scaled Chebyshev coefficients and their limit superior). If $b_{n}$ are the Chebyshev coefficients of a function, then $\tilde{b}_{n}$ are the Chebyshev coefficients scaled by the optimal envelope function for $b_{n}$. In other words,

$$
\tilde{b}_{n} \equiv \frac{b_{n}}{E(n)} .
$$

Thus, the following properties hold:

1. (upper bound) $\left|\tilde{b}_{n}\right| \leq 1$ for all $\mathrm{n}$.

2. (infinite number of terms in sequence that are arbitrarily close to one) For any $\delta>0$, there exists a subset of $\left|\tilde{b}_{n}\right|$, called $g_{n}$, with an infinite number of terms such that $g_{n}>1-\delta$.

3. $\lim \sup _{n \rightarrow \infty}\left|\tilde{b}_{n}\right|=1$.

Definition 4.3 (Scaled differences). If $b_{n}$ are the Chebyshev coefficients of a function, then the Chebyshev coefficients of the first integral of the function are given by

$$
c_{n}=\frac{1}{2 n}\left(b_{n-1}-b_{n+1}\right) .
$$

The scaled differences, $\tilde{\gamma}_{n}$, are then defined as

$$
\begin{aligned}
\tilde{\gamma}_{n} & \equiv \frac{b_{n-1}-b_{n+1}}{E(n-1)} \\
& \equiv\left\{\tilde{b}_{n-1}-\frac{E(n+1)}{E(n-1)} \tilde{b}_{n+1}\right\} .
\end{aligned}
$$

The following definition is used as a criterion for determining the strength of the upper bound given in Theorem 4.1.

Definition 4.4 (Strongly self-cancelling coefficients). The Chebyshev series coefficients are "strongly self-cancelling" if

$$
\limsup _{n \rightarrow \infty}\left|\tilde{\gamma}_{n}\right|=0 .
$$

Conjecture 4.5. Only if the Chebyshev coefficients are "strongly self-cancelling", as defined above, is the inequality of Theorem 4.1 weakened.

The reason for this conjecture will become evident in the second example given in Section 5. However, it can be seen from (4.12) that if $b_{n}$ is converging slowly, in other words algebraically (refer to Chapter 2 of [6]), then in a Taylor series 
expansion of the coefficients $b_{n-1}$ would cancel $b_{n+1}$ to lowest order, contributing an extra factor of $O\left(\frac{1}{n}\right)$. In such a case, the theorem overestimates the coefficients of the integral by $O(n)$.

To prove that Definition 4.4 does not hold in the case when the Chebyshev coefficients of a function are converging geometrically, we need the following definition and two lemmas.

Definition 4.6. A Chebyshev series with coefficients $b_{n}$ is said to have a "geometric" rate of convergence if

$$
\limsup _{n \rightarrow \infty} \frac{E(n+1)}{E(n-1)}=\frac{1}{\rho^{2}}<1,
$$

where $\rho$ is a constant that is greater than 1 and $E\left(n ;\left\{b_{n}\right\}\right)$ is an optimal envelope function $\left\{b_{n}\right\}$. Note that if, for example, $E(n) \equiv \frac{1}{\rho^{n}} n^{k}$, then

$$
\frac{E(n+1)}{E(n-1)}=\frac{1}{\rho^{2}}\left\{1+\frac{2 k}{n}+O\left(\frac{1}{n^{2}}\right)\right\} \text {. }
$$

This definition is consistent with the alternative definition given in [6]. "Supergeometric" convergence (e.g. $E(n)=\frac{1}{n^{n}} n^{k}$ ) is the limit as $\rho \rightarrow \infty$.

The next two lemmas were proved in [2].

Lemma 4.7. Given two real-valued sequences $\left\{a_{n}\right\}$ and $\left\{b_{n}\right\}$ bounded below, then

$$
\limsup _{n \rightarrow \infty}\left(a_{n}-b_{n}\right) \geq \limsup _{n \rightarrow \infty} a_{n}-\limsup _{n \rightarrow \infty} b_{n} .
$$

Lemma 4.8. If $a_{n}>0$ and $b_{n}>0$ for all $n$, and if the $\limsup _{n \rightarrow \infty} a_{n}$ and $\limsup _{n \rightarrow \infty} b_{n}$ are finite or both are infinite, then

$$
\limsup _{n \rightarrow \infty}\left(a_{n} b_{n}\right) \leq\left(\limsup _{n \rightarrow \infty} a_{n}\right)\left(\limsup _{n \rightarrow \infty} b_{n}\right) .
$$

Theorem 4.9. If the Chebyshev coefficients of a function are converging geometrically then

$$
\left(1-\frac{1}{\rho^{2}}\right) \leq \limsup _{n \rightarrow \infty}\left|\tilde{\gamma}_{n}\right|=\limsup _{n \rightarrow \infty}\left|2 n \frac{c_{n}}{E(n-1)}\right| \leq\left(1+\frac{1}{\rho^{2}}\right),
$$

where $\tilde{\gamma}_{n}$ and $\rho$ are defined above and $c_{n}$ are the coefficients of the first integral of the function as given in Definition 4.3.

Proof. The left hand side of (4.20) is proved as follows:

$$
\begin{aligned}
\tilde{\gamma}_{n} & =\left\{\tilde{b}_{n-1}-\frac{E(n+1)}{E(n-1)} \tilde{b}_{n+1}\right\} \\
\left|\tilde{\gamma}_{n}\right| & \geq\left\{\left|\tilde{b}_{n-1}\right|-\left|\frac{E(n+1)}{E(n-1)}\right|\left|\tilde{b}_{n+1}\right|\right\}, \\
\limsup _{n \rightarrow \infty}\left|\tilde{\gamma}_{n}\right| & \geq \limsup _{n \rightarrow \infty}\left\{\left|\tilde{b}_{n-1}\right|-\left|\frac{E(n+1)}{E(n-1)}\right|\left|\tilde{b}_{n+1}\right|\right\} \\
& \geq \limsup _{n \rightarrow \infty}\left|\tilde{b}_{n-1}\right|-\limsup _{n \rightarrow \infty}\left|\frac{E(n+1)}{E(n-1)}\right|\left|\tilde{b}_{n+1}\right| \\
& \geq \limsup _{n \rightarrow \infty}\left|\tilde{b}_{n-1}\right|-\limsup _{n \rightarrow \infty}\left|\frac{E(n+1)}{E(n-1)}\right| \limsup _{n \rightarrow \infty}\left|\tilde{b}_{n+1}\right| \\
& \geq 1-\frac{1}{\rho^{2}}
\end{aligned}
$$


The right hand side of (4.20) is proved in exactly the same manner except the inequality is reversed since $\left|\tilde{\gamma}_{n}\right| \leq\left\{\left|\tilde{b}_{n-1}\right|+\left|\frac{E(n+1)}{E(n-1)}\right|\left|\tilde{b}_{n+1}\right|\right\}$.

\section{EXAMPLES}

In this section, two examples are given. The first serves two purposes: 1) to show that indeed Theorem 4.1 gives the optimal envelope function as $n \rightarrow \infty$ for the first integral of a function whose Chebyshev series is converging geometrically, and 2) to demonstrate the usefulness of such a theorem in cases where applying the method of steepest descent for determining the asymptotic behavior of the coefficients is laborious. The second example gives a case where the upper bound given by Theorem 4.1 is weakened. An analysis is done to show what class of functions causes the weakening of the theorem and why.

5.1. Case : A function with a super-geometrically converging Chebyshev series. As an example, the asymptotic behavior of the coefficients of both a Gaussian centered at an endpoint of the Chebyshev expansion interval and its first integral, the error function, will be considered for large $n$. This example was chosen for two reasons: 1) the application of the method of steepest descent is both tedious and laborious, and 2) it has not been previously given in the literature. The method itself can be found in standard advanced texts in applied mathematics [3]. It finds the asymptotic behavior of an integral of the form

$$
I(n)=\int_{C} g(t) e^{\phi(t, n)} d t
$$

as $n \rightarrow \infty$, where $\mathrm{C}$ is a contour in the complex plane. The first step is to deform the path of integration such that the main contribution to the integral as $n \rightarrow \infty$ comes from the neighborhood of the points where the argument of the exponential, $\phi$, has a saddle point. These points are called stationary points, $t_{s}(n)$, and are found by solving

$$
\frac{d \phi\left(t_{s}, n\right)}{d t}=0
$$

Then

$$
I(n) \sim \sum \sqrt{\frac{2 \pi}{-\phi_{t t}\left(t_{s}\right)}} e^{\phi\left(t_{s}, n\right)} g\left(t_{s}\right),
$$

where the summation is over all stationary points that lie on the path of integration.

The series expansion for a Gaussian centered at the endpoint $x=-1$ of the Chebyshev interval $[-1,1]$ is given by

$$
\begin{aligned}
e^{-A(x+1)^{2}} & =\sum_{n=0}^{\infty} a_{n} T_{n}(x) \\
\text { (5.4) } \quad a_{n} & =\frac{1}{\pi}\left\{\int_{0}^{\pi} e^{-A(\cos (t)+1)^{2}+i n t} d t+\int_{0}^{\pi} e^{-A(\cos (t)+1)^{2}-i n t} d t\right\}, n>0,
\end{aligned}
$$

where $A$ is a parameter determining the width of the Gaussian and the transformation $x=\cos (t)$ has been used. The method of steepest descent gives the 
approximation

$$
a_{n}=\operatorname{Re}\left\{\sqrt{\frac{8}{-\pi \phi_{t t}\left(t_{s}\right)}} e^{-A\left(\cos \left(t_{s}\right)+1\right)^{2}+i n t_{s}}\right\} .
$$

We find $t_{s}$ by solving

$$
\frac{d \phi}{d t}=\sin (2 t)+2 \sin (t)+i \frac{n}{A}=0
$$

which gives

$$
t_{s}=2 \arctan (z)
$$

where $z$ is a root of the quartic equation

$$
z^{4}+z^{2}+1-\frac{A}{n} 8 i z=0
$$

In this case, only a single stationary point lies on the deformed path of integration and contributes to the approximation of the integral. This corresponds to the root of equation 5.8 for which both the real and imaginary parts are positive. In order to get an analytical expression for equation 5.5 in terms of $n$, a perturbation analysis to the first order is done on equation 5.8 with $\frac{A}{n}$ treated as a small parameter for $n \rightarrow \infty$ and $A$ fixed. It is important to remember that the rate of convergence associated with the approximation is controlled by the real part of $\phi\left(t_{s}(n), n\right)$. Theorem 1 of [4] states that the change in the real part of $\phi\left(t_{s}(n), n\right)$ with $n$ is given by the negative of the imaginary part of the stationary point, $t_{s}$; in other words,

$$
\Re\left(\frac{d \phi\left(t_{s}(n), n\right)}{d n}\right)=-\Im\left(t_{s}(n)\right) .
$$

In the example of the Gaussian, the real part of the contributing stationary point approaches zero and the imaginary part approaches $\infty$ as $n \rightarrow \infty$. This is the distinguishing characteristic of "super-geometric convergence" [6], that the imaginary part of the stationary point increases with $n$. Thus, the combined use of the theorem with trigonometric identities yields the expected super-geometric convergence for the Chebyshev coefficients of a Gaussian,

$$
\begin{aligned}
a_{n} \sim \alpha & \cos \left(\left(n+\frac{A}{2}\right) \arctan \left(\sqrt{\frac{2 n}{A}}\right)-\frac{A}{2} \sqrt{\frac{2 n}{A}}\right) \\
& \exp \left(-\frac{n}{2} \ln \left(1+\frac{2 n}{A}\right)-\frac{A}{4} \ln \left(1+\frac{2 n}{A}\right)+\frac{A}{4}+\frac{n}{2}\right) \quad \text { for large } \frac{n}{A},
\end{aligned}
$$

where $\alpha$ denotes a term which varies only algebraically (not exponentially) with $n$. This term is the contribution from $\sqrt{\frac{8}{-\pi \phi_{t t}\left(t_{s}\right)}}$ and is given by

$$
\begin{aligned}
\alpha & =\sqrt{\frac{8}{-\pi}}\left(4 \cos ^{2}\left(\arctan \left(\sqrt{\frac{2 n}{A}}\right)\right) \cosh ^{2}\left(\frac{1}{2} \ln \left(1+\frac{2 n}{A}\right)\right)\right. \\
& -4 \sin ^{2}\left(\arctan \left(\sqrt{\frac{2 n}{A}}\right)\right) \sinh ^{2}\left(\frac{1}{2} \ln \left(1+\frac{2 n}{A}\right)\right) \\
& \left.+4 \cos \left(\arctan \left(\sqrt{\frac{2 n}{A}}\right)\right) \cosh \left(\frac{1}{2} \ln \left(1+\frac{2 n}{A}\right)\right)-2\right)^{-\frac{1}{2}}
\end{aligned}
$$


(a)

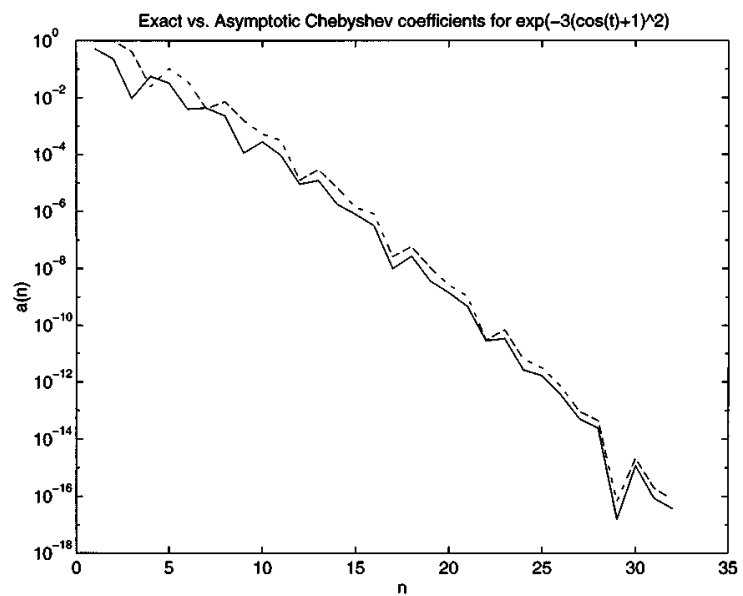

(b)

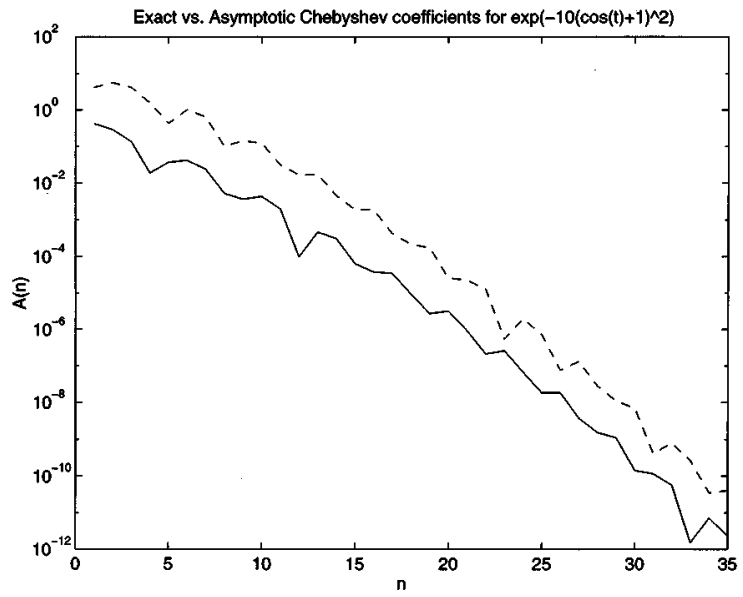

(c)

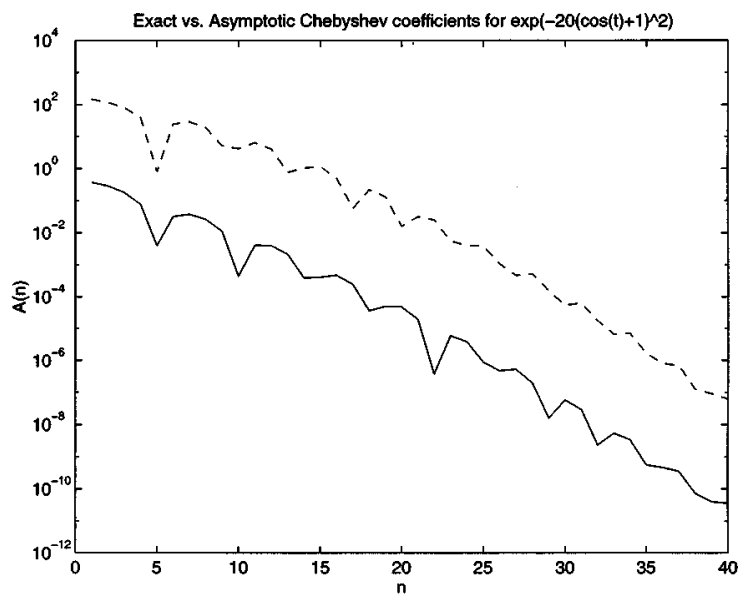

Figure 1. Solid: Exact Chebyshev coefficients for $\exp \left(-A(x+1)^{2}\right)$. Dashed: The full asymptotic approximation given by (5.10) (a) $\mathrm{A}=3$ (b) $\mathrm{A}=10$ (c) $\mathrm{A}=20$. 
The full asymptotic approximation to the Chebyshev coefficients versus the exact coefficients for the Gaussian centered at the endpoint of the expansion interval can be seen in Figure 1 for $A=3, A=10$, and $A=20$. The asymptotic relation captures the general exponential trend of decay of the coefficients. However, as $A$ increases, the error between the asymptotic and exact coefficients increases by a multiplicative factor. There are two sources of error in the approximation of the coefficients: 1) the method of steepest descent is only being carried out to the lowest order, and 2) the solution to equation 5.8 is being determined only to the first order. Since the stationary point is approximated by perturbation theory with $A / n$ treated as a small parameter, the asymptotic relation given by (5.10) breaks down for large $A$ and fixed $n$. Thus, as $A$ becomes larger, the relative error increases. Although not shown here, the error would be orders of magnitude smaller if equation 5.8 were solved numerically rather than through perturbation theory. However, this would defeat the purpose of deriving an asymptotic relation as opposed to numerically calculating the exact coefficients. Furthermore, there are two counteracting forces at play. As $A$ increases, $A / n$ becomes larger, eventually rendering the perturbative solution to equation 5.8 useless. On the other hand, the rate at which the error decreases with $n$ slows as $A$ becomes larger. This is due to the fact that a narrower Gaussian introduces steepest gradients requiring more Chebyshev polynomials to evaluate it to a given accuracy. The restriction to $A / n$ small might be circumvented by deriving a uniform asymptotic expansion in the spirit of [5], but is not done here. It should also be noted that for a Gaussian centered in the middle of the expansion interval the Chebyshev coefficients are proportional to $I_{n}\left(\frac{A}{2}\right)$ [7]. For small values of $A$, that is for very wide Gaussians, the asymptotic rate of convergence of the coefficients is essentially the same as for the Gaussian centered at the endpoint. However, as $A$ increases the coefficients for the endpoint centered Gaussian converge much faster because the Chebyshev polynomials oscillate more rapidly near the endpoints leading to a higher effective resolution (refer to [6], Chapter 2).

If we are interested in finding the asymptotic behavior as $n \rightarrow \infty$ for the Chebyshev coefficients of an error function, the usual way to proceed is to apply the method of steepest descent to the integral representation of the coefficients. As seen above, this can be an involved and laborious procedure. Having done the asymptotic analysis for the Gaussian, we can manipulate Theorem 2.1 to find the exact Chebyshev coefficients of its first integral, the error function. These coefficients are given by

$$
\frac{1}{2 n}\left(a_{n-1}-a_{n+1}\right)
$$

where $a_{n}$ is defined in (5.10). However, due to the complicated nature of (5.10), evaluating (5.12) is not easy. A simpler, more immediate interpretation for the asymptotic behavior of the Chebyshev coefficients for the error function is provided by Theorem 4.1. The optimal envelope function for the Chebyshev coefficients of

$$
\frac{\sqrt{\pi}}{2 \sqrt{A}} \operatorname{erf}(\sqrt{A}(x+1))
$$

the first integral of $\exp \left(-A(x+1)^{2}\right)$ as $n \rightarrow \infty$ for fixed $A$, is simply the optimal envelope function for the Chebyshev coefficients of the Gaussian with argument 


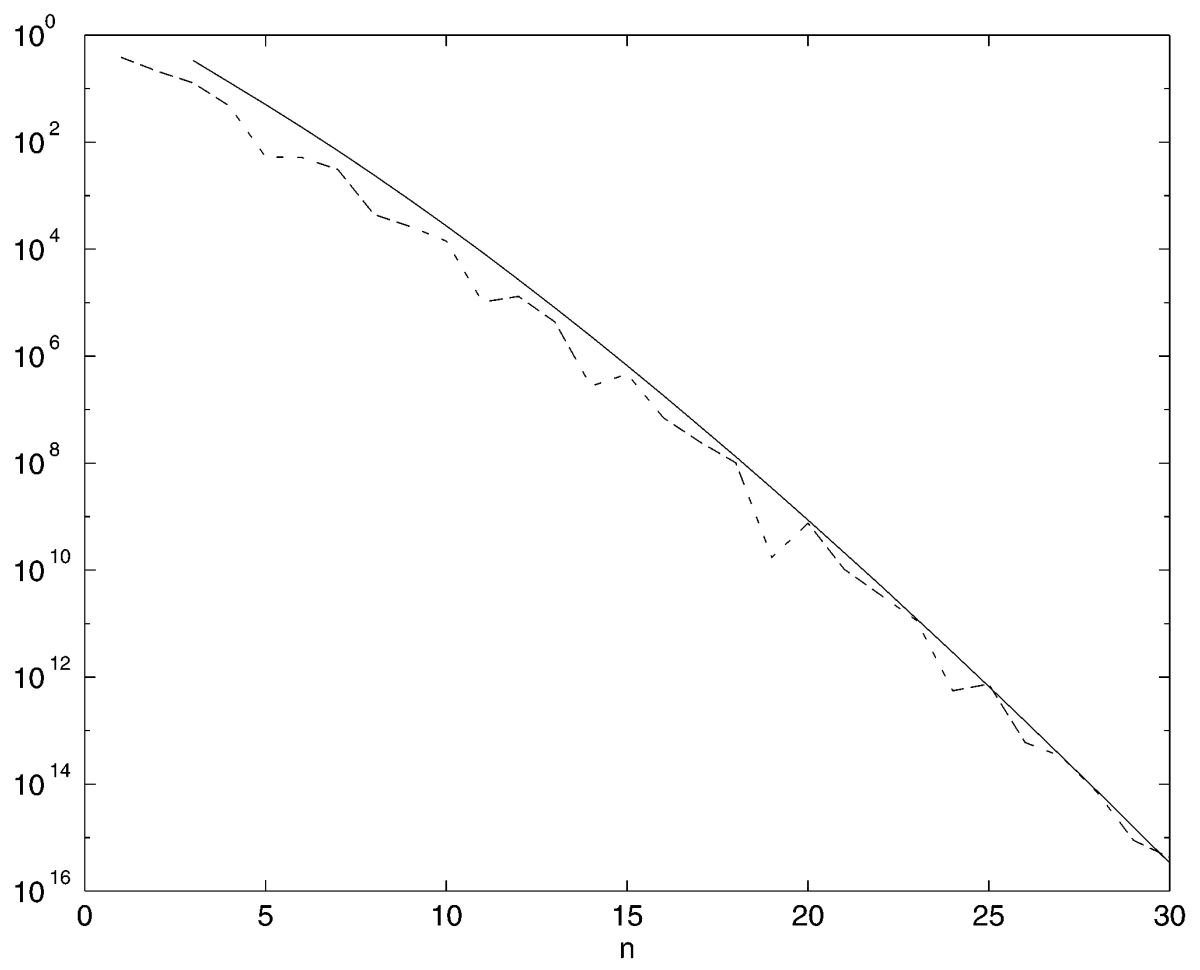

Figure 2. Dashed: Exact Chebyshev coefficients for $\frac{\sqrt{\pi}}{2 \sqrt{A}} \operatorname{erf}(\sqrt{A}(x+1))$ with $A=3$. Solid: Optimal envelope function given by (5.13).

$(n-1)$ divided by $n$. In other words,

$$
\begin{aligned}
E_{n}^{(e r f)} \sim \frac{1}{n}\{\beta \exp ( & -\frac{(n-1)}{2} \ln \left(1+\frac{2(n-1)}{A}\right) \\
& \left.\left.-\frac{A}{4} \ln \left(1+\frac{2(n-1)}{A}\right)+\frac{A}{4}+\frac{(n-1)}{2}\right)\right\},
\end{aligned}
$$

where $\beta$ is defined in (5.11) with the $n$ being replaced by $(n-1)$. Figure 2 shows the envelope function given by (5.13) versus the exact coefficients for $\frac{\sqrt{\pi}}{2 \sqrt{A}} \operatorname{erf}(\sqrt{A}(x+1))$ calculated numerically.

5.2. Case 2: A function with an algebraically converging Chebyshev series. As an example of where the coefficients are "strongly self-cancelling", given in Definition 4.4, we will consider the function whose Chebyshev coefficients decrease as

$$
b_{n} \sim \frac{1}{n^{j}},
$$

where $j$ is a constant greater than 1 . If we define the optimal envelope function for $b_{n}$ by (5.14) then according to Theorem 4.1, the Chebyshev coefficients $c_{n}$ for the 


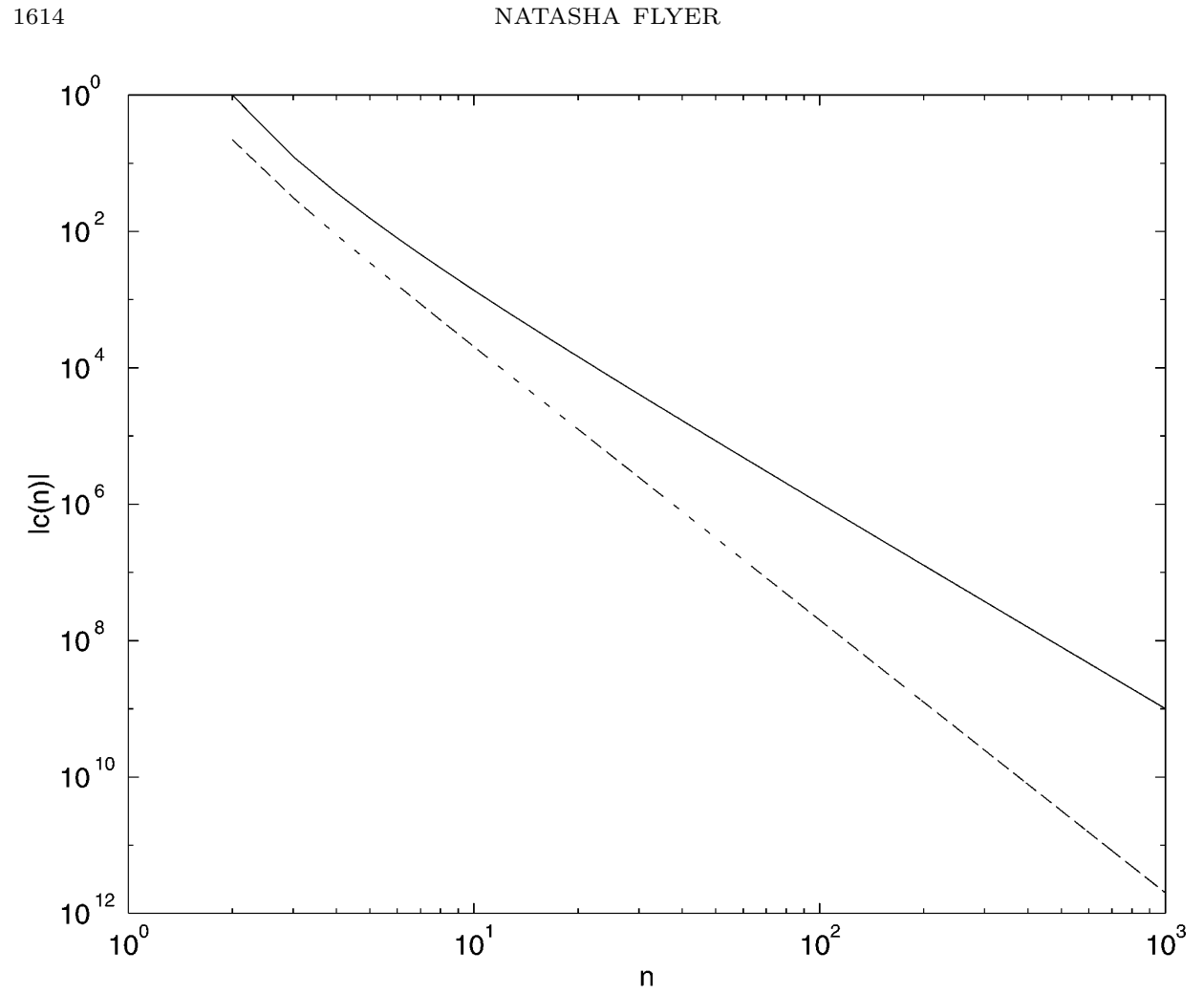

Figure 3. Dashed: Exact Chebyshev coefficients given by $\frac{1}{2 n}\left(b_{n-1}-b_{n+1}\right)$ where $b_{n} \sim \frac{1}{n^{2}}$. Solid: The upper bound given by Theorem 4.1.

first integral of the function are bounded from above to lowest order by

$$
\left|c_{n}\right| \leq \frac{1}{n(n-1)^{j}}=\frac{1}{n^{j+1}\left(1-\frac{1}{n}\right)^{j}}=\frac{1}{n^{j+1}}+O\left(\frac{1}{n^{j+2}}\right) .
$$

Figure 3 shows the case for $j=2$. It can indeed be seen that (5.15) gives a loose upper bound on the exact coefficients for $c_{n}$, which worsens as $n$ grows larger. A little analysis will show why this is the case as well as enforce the conjecture made in Section 2 as to the weakening of Theorem 4.1 with regard to "strongly self-cancelling" Chebyshev coefficients.

We know that the exact coefficients for the integral of the function are given by

$$
c_{n}=\frac{1}{2 n}\left\{\frac{1}{(n-1)^{j}}-\frac{1}{(n+1)^{j}}\right\} .
$$

If the series were converging rapidly, say geometrically, the first term in (5.16) would dominate and we would essentially have the upper bound given by (5.15). However, the coefficients of $b_{n}$ are "strongly self-cancelling" in the sense of Definition 4.4. This 
can be seen directly by expanding (5.16) in terms of a Taylor series,

$$
\begin{aligned}
c_{n} & =\frac{1}{2 n}\left\{\frac{1}{(n-1)^{j}}-\frac{1}{(n+1)^{j}}\right\} \\
& =\frac{1}{2 n^{j+1}}\left\{\frac{1}{\left(1-\frac{1}{n}\right)^{j}}-\frac{1}{\left(1+\frac{1}{n}\right)^{j}}\right\} \\
& =\frac{1}{2 n^{j+1}}\left\{1+\frac{j}{n}-1+\frac{j}{n}+O\left(\frac{1}{n^{2}}\right)\right\} \\
& =\frac{1}{n^{(j+1)+1}}+O\left(\frac{1}{n^{(j+2)+1}}\right) .
\end{aligned}
$$

We see to lowest order $b_{n-1}$ cancels $b_{n+1}$, leading to an order of convergence that is greater by a factor of $\frac{1}{n}$ than that given by (5.15). (5.20) is an analysis of $\tilde{\gamma}_{n}$. Since $\tilde{b}_{n-1}=\tilde{b}_{n+1}=1$ and $\frac{E(n+1)}{E(n-1)}=1+O\left(\frac{1}{n}\right)$, we have

$$
\left|\tilde{\gamma}_{n}\right|=\left|\left\{1-\left(1+O\left(\frac{1}{n}\right)\right)\right\}\right| .
$$

Thus,

$$
\limsup _{n \rightarrow \infty}\left|\tilde{\gamma}_{n}\right|=0
$$

It is for the reasons above that the conjecture in Section 2 was made. Furthermore, we make the conjecture that only functions which are regular everywhere on $x \epsilon[-1,1]$, except at the endpoints of the Chebyshev interval $x=1$ and $x=-1$, will have this "strongly self-cancelling" effect of the coefficients resulting in an order of convergence that is faster by a factor of $\frac{1}{n}$ than Theorem 4.1 would imply. This conjecture is backed up by the work of Elliott [8] who shows that functions which are regular except at $x= \pm 1$ (e.g. $f(x)=(1 \pm x)^{\phi} g(x)$, where $\phi$ is not an integer and $g(x)$ is regular everywhere on $x \epsilon[-1,1]$ including the endpoints) have Chebyshev coefficients $c_{n} \simeq O\left(\frac{1}{n^{2 \phi+1}}\right)$. Therefore the difference between the function and its integral is $O\left(\frac{1}{n^{2}}\right)$ as can be seen from the simple example $g(x)=1$ and $\phi=\frac{1}{2}$. However, Theorem 4.1 implies that the difference should be $O\left(\frac{1}{n}\right)$, thus predicting decay which is too slow by $O\left(\frac{1}{n}\right)$. This is the behavior the theorem exhibits when the coefficients are "strongly self-cancelling". Thus, it is conjectured that Theorem 4.1 gives the tightest upper bound possible as $n \rightarrow \infty$ for the absolute value of the Chebyshev coefficients of the first integral of a function not only for series whose coefficients are geometrically converging but for all functions except those that have singularities on $x \epsilon[-1,1]$ exclusively at $x= \pm 1$. This conjecture is made since the increase in convergence by the extra factor of $\frac{1}{n}$ as seen by the functions in Elliot's paper is believed to be solely an endpoint effect.

\section{Summary}

The importance of this paper is that we were able to derive the asymptotic relationship between the optimal envelope function that bounds the Chebyshev coefficients from above and the optimal envelope function for the coefficients of the integral of the function. First, a theorem was developed that provides an upper bound to the Chebyshev coefficients of the $k^{t h}$ integral of a function. The question then asked was: How tight is this upper bound? In order to determine a criterion under which the theorem did not give an optimal upper bound as $n \rightarrow \infty$, we specialized to the case $k=1$. It was conjectured then that only if the Chebyshev 
coefficients of a function were "strongly self-cancelling", as defined above, does the theorem not provide the tightest upper bound possible, i.e. the optimal envelope function. This conjecture was based on the fact that coefficients which obeyed the criterion cancelled each other to the lowest order, resulting in a contribution of an extra factor of $\frac{1}{n}$. Functions that are regular on the interval $[-1,1]$ except at the endpoints have these types of coefficients. Furthermore, it was shown that in geometrically converging Chebyshev series, which is the usual rate of convergence, the coefficients are not "strongly self-cancelling" and the theorem provided the optimal envelope function for the coefficients as $n \rightarrow \infty$. In such cases, the theorem provides a good alternative to the often laborious method of steepest descent. In the last section, two examples were given to illustrate these concepts. The first example considers a function whose Chebyshev series converges super-geometrically, a Gaussian and its first integral the error function. The second example considers a function and its first integral whose Chebyshev series converges algebraically, a function that is regular except at the endpoints $x= \pm 1$ of the Chebyshev interval $x \in[-1,1]$.

However, more work needs to be done. It would be beneficial to prove that if the Chebyshev coefficients of a function are converging as $\frac{1}{n^{j+1}}$ or $\frac{1}{n^{j+1}}(-1)^{n}$, where $j$ is a constant greater than zero, then the function is singular exclusively at the endpoints $x= \pm 1$ of the Chebyshev interval. Secondly, it is believed that the same results should hold for higher integrals, that is for $k=2,3, \ldots$ and so forth. The reason is that the coefficients for the higher integrals of a function are just weighted combinations of the coefficients of the original function in the form of an alternating series.

\section{REFERENCES}

1. M. Abramowitz and I.A. Stegun Handbook of Mathematical Functions, Dover, New York, 1972. MR 94b:00012

2. T.M. Apostol Mathematical Analysis, Addison-Wesley, Reading, Massachusetts, 1974. MR 49:9123

3. C.M. Bender and S. Orszag, Advanced Mathematical Methods for Scientists and Engineers, McGraw-Hill, New York, 1978. MR 80d:00030

4. J.P. Boyd, The rate of convergence of Fourier coefficients for entire functions of infinite order with application to the Weideman-Cloot sinh-mapping for pseudospectral computations on an infinite interval, J. Comp. Phys. vol. 110, 1994, pp. 360-375. CMP 94:10

5. J.P. Boyd, Asymptotic coefficients of Hermite Function Series, J. Comp. Phys. vol. 54, 1984, pp. 382-410. MR 86c: 41012

6. J.P. Boyd, Chebyshev and Fourier Spectral Methods, Springer-Verlag, New York, 1989.

7. D. Elliott and G. Szekeres, Some estimates of the coefficients in the Chebyshev series expansion of a function Math. Comp. vol. 19, 1965, pp. 25-32. MR 30:2666

8. D. Elliott, The evaluation and estimation of the coefficients in the Chebyshev series expansion of a function Math. Comp. vol. 18, 1964, pp. 274-281. MR 29:4176

9. L. Fox and I.B. Parker, Chebyshev Polynomials in Numerical Analysis, Oxford University Press, London, 1968. MR 37:3733

Department of Atmospheric, Oceanic, and Space Sciences, University of Michigan, Ann Arbor, Michigan 48105

E-mail address: nflyer@engin.umich.edu 